\title{
Intersections of Amoebas
}

\author{
Martina Juhnke-Kubitzke卯 and Timo de Wolff" \\ ${ }^{1}$ Universität Osnabrück, Germany \\ ${ }^{2}$ Texas A\&M University, USA
}

\begin{abstract}
Amoebas are projections of complex algebraic varieties in the algebraic torus under a Log-absolute value map, which have connections to various mathematical subjects. While amoebas of hypersurfaces have been intensively studied during the last years, the non-hypersurface case is barely understood so far. We investigate intersections of amoebas of $n$ hypersurfaces in $\left(\mathbb{C}^{*}\right)^{n}$, which are genuine supersets of amoebas given by non-hypersurface varieties. Our main results are amoeba analogs of Bernstein's Theorem and Bézout's Theorem providing an upper bound for the number of connected components of such intersections. Moreover, we show that the order map for hypersurface amoebas can be generalized in a natural way to intersections of amoebas. We show that, analogous to the case of amoebas of hypersurfaces, the restriction of this generalized order map to a single connected component is still 1-to-1.

Résumé. Les amibes sont la projection de variétés algébriques complexes par la fonction Log-module. Elles sont en liens avec de nombreuses branches des mathématiques. Alors que les amibes d'hypersurfaces ont été intensivement étudiées ces dernières années, on comprend encore très peu le cas de la codimension supérieur à 1 . Nous nous intéressons à l'intersection de $n$ amibes d'hypersurfaces dans $\left(\mathbb{C}^{*}\right)^{n}$. C'est un sur-ensemble strict de l'amibe de l'intersections des $n$ hypersurfaces. Notre résultat principal est analogue aux Théorèmes de Berstein et de Bézout donnant une borne supérieure au nombre de composantes connexes de telles intersections. De plus, nous montrons que la fonction d'ordre d'amibes d'hypersurfaces peut être généralisée de manière naturelle à l'intersection d'amibes. Nous montrons que de manière analogue au cas d'amibes d'hypersurfaces la restriction de cette fonction d'ordre généralisée à une composante connexe est également injective.
\end{abstract}

Keywords. Amoeba, Bernstein's Theorem, Intersection, Mixed Volume, Order Map, Tropical Geometry

\section{Introduction}

Let $I$ be an ideal generated by Laurent polynomials $f_{1}, \ldots, f_{k} \in \mathbb{C}\left[\mathbf{z}^{ \pm 1}\right]:=\mathbb{C}\left[z_{1}^{ \pm 1}, \ldots, z_{n}^{ \pm 1}\right]$ and let $\mathcal{V}(I) \subseteq\left(\mathbb{C}^{*}\right)^{n}:=(\mathbb{C} \backslash\{0\})^{n}$ be the corresponding variety. The amoeba $\mathcal{A}(I)$ of $I$, as originally defined by Gelfand, Kapranov, and Zelevinsky Gelfand et al. (1994), is the image of $\mathcal{V}(I)$ under the Log-absolute map given by

$$
\log |\cdot|:\left(\mathbb{C}^{*}\right)^{n} \rightarrow \mathbb{R}^{n}, \quad\left(z_{1}, \ldots, z_{n}\right) \mapsto\left(\log \left|z_{1}\right|, \ldots, \log \left|z_{n}\right|\right) .
$$

\footnotetext{
${ }^{\dagger}$ Email: juhnke-kubitzke@uni-osnabrueck.de. MJ was supported by the German Research Council DFGGRK 1916.

‡Email: dewolff@math. tamu.edu TdW was partially supported by GIF Grant no. 1174/2011, DFG project MA 4797/3-2 and DFG project TH 1333/2-1.
}

1365-8050 (c) 2016 Discrete Mathematics and Theoretical Computer Science (DMTCS), Nancy, France 
In the special case that $I=\langle f\rangle$, we write $\mathcal{A}(f)$ for simplicity.

Amoebas became prominent during the last twenty years since they provide a natural connection between algebraic geometry and tropical geometry; see de Wolff (2015); Maclagan and Sturmfels (2015); Mikhalkin (2004) for an overview. Furthermore, amoebas are objects with a rich structure themselves and there exist connections to numerous other mathematical subjects like complex analysis Forsberg et al. (2000), nonnegativity of real polynomials Iliman and de Wolff|(2014), crystal shapes Kenyon et al. (2006), the topology of real curves Mikhalkin (2000), and statistical thermodynamics Passare et al. (2012).

Though amoebas of hypersurfaces have been intensively studied during the last years, the non-hypersurface case is still barely understood. For almost all properties that are true in the hypersurface case it is not known if they still hold for arbitrary varieties. One of the few exceptions was shown by Purbhoo. It states that the amoeba of an arbitrary ideal $I \subseteq \mathbb{C}\left[\mathbf{z}^{ \pm 1}\right]$ can be written as the intersection of the amoebas of all the elements of $I$; see (Purbhoo, 2008, Corollary 5.6.):

$$
\mathcal{A}(I)=\bigcap_{f \in I} \mathcal{A}(f)
$$

Since this result is not useful from a computational point of view, the question about the existence of an amoeba basis arose in the article Schroeter and de Wolff](2013) by Schroeter and the second author. Here, an amoeba basis refers to a finite set of Laurent polynomials $f_{1}, \ldots, f_{k}$ such that $\left\langle f_{1}, \ldots, f_{k}\right\rangle=I$ and $\mathcal{A}(I)=\bigcap_{j=1}^{k} \mathcal{A}\left(f_{k}\right)$. So far, amoeba bases are known to exist only in very special cases Schroeter and de Wolff (2013), while a recent result by Nisse claims non-existence in general Nisse (2014). By [1.2], the inclusion $\mathcal{A}(I) \subseteq \bigcap_{j=1}^{k} \mathcal{A}\left(f_{k}\right)$ holds for every collection $f_{1}, \ldots, f_{k}$ with $\left\langle f_{1}, \ldots, f_{k}\right\rangle \subseteq I$ and it is reasonable to expect that information about $\bigcap_{j=1}^{k} \mathcal{A}\left(f_{k}\right)$ also provides information about $\mathcal{A}(I)$. Phrased differently, understanding finite intersections of hypersurface amoebas is an essential interim stage for understanding amoebas of arbitrary ideals. This serves as the key motivation for this article.

Let $\mathcal{F}:=\left\{f_{1}, \ldots, f_{n}\right\} \subseteq \mathbb{C}\left[\mathbf{z}^{ \pm 1}\right]$ be a family of $n$-variate complex Laurent polynomials. We study the intersection of their corresponding amoebas $\mathcal{A}\left(f_{1}\right), \ldots, \mathcal{A}\left(f_{n}\right) \subseteq \mathbb{R}^{n}$. We show that intersections $\mathcal{I}(\mathcal{F}):=\bigcap_{j=1}^{n} \mathcal{A}\left(f_{j}\right)$ preserve a significant amount of the amoeba structure from the hypersurface case. Moreover, intersections $\mathcal{I}(\mathcal{F})$ carry a rich combinatorial structure.

Since, in general, $\mathcal{I}(\mathcal{F})$ consists of several connected components, we focus on the study of the combinatorics of these components; see Figure 1 and Section 3 Particularly, the convex hull of each of these components is proven to be a simple polytope, which, in the sequel, will be referred to as intersection polytope. One of our main results provides an upper bound for the number of connected components of an intersection $\mathcal{I}(\mathcal{F})$. More precisely, as an analog to the classical Bernstein's Theorem, we show the following Amoeba Bernstein Theorem; see Theorem 3.10 .

Theorem 1.1 Let $\mathcal{F}=\left\{f_{1}, \ldots, f_{n}\right\} \subseteq \mathbb{C}\left[\mathbf{z}^{ \pm 1}\right]$ be a generic collection of Laurent polynomials. The number of connected components of $\mathcal{I}(\mathcal{F})$ is bounded from above by the mixed volume $\operatorname{MV}\left(\operatorname{New}\left(f_{1}\right), \ldots, \operatorname{New}\left(f_{n}\right)\right)$.

We specify in Section 3 , what we understand by a "generic collection of Laurent polynomials". We remark that this theorem is highly non-obvious since it is unclear whether every connected component of $\mathcal{I}(\mathcal{F})$ contains a point, which is the projection of a point in $\mathcal{V}\left(\left\langle f_{1}, \ldots, f_{n}\right\rangle\right)$ with respect to the $\log |\cdot|-$ map. As a consequence we obtain the following Amoeba Bézout Theorem; see Theorem 3.11 . 


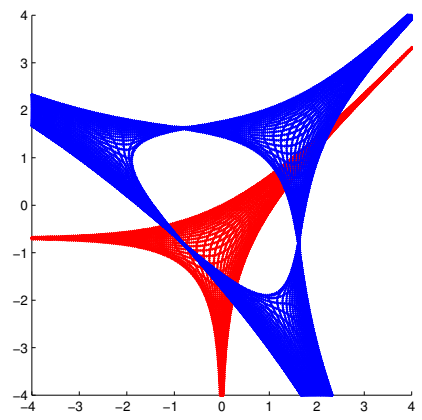

Fig. 1: An approximation of the amoebas $\mathcal{A}\left(f_{1}\right)$ and $\mathcal{A}\left(f_{2}\right)$ for $f_{1}:=2 z_{1}+z_{2}+1$ and $f_{2}:=z_{1}^{2} z_{2}+z_{1} z_{2}^{2}+5 z_{1} z_{2}+1$. The intersection $\mathcal{A}\left(f_{1}\right) \cap \mathcal{A}\left(f_{2}\right)$ consists of two connected components.

Theorem 1.2 Let $\mathcal{F}=\left\{f_{1}, \ldots, f_{n}\right\} \subseteq \mathbb{C}\left[\mathbf{z}^{ \pm 1}\right]$ be a generic family of Laurent polynomials. The number of connected components of $\mathcal{I}(\mathcal{F})$ is smaller than or equal to the product of the total degrees of the $f_{j}$.

In Section 4, we construct a generalization of the order map of hypersurface amoeba. The classical order map (see Forsberg et al. (2000)) relates the components of the complement of a hypersurface amoeba $\mathcal{A}(f)$ to the lattice points in the corresponding Newton polytope New $(f)$; see Section 2.2 for details. Given a collection $\mathcal{F}$ of $n$ Laurent polynomials, we define a natural generalization of the order map to the vertex sets of the intersection polytopes of $\mathcal{F}$ and thereby also to the polytope, which is the convex hull of the entire intersection $\mathcal{I}(\mathcal{F})$. We show that several properties of the order map for the hypersurface case are preserved in this more general setting; see Theorems 4.1 and 4.4 as well as Corollary 4.5. The next theorem summarizes those results. The notion of a mixed normal cone, which is used in this statement, will be explained in Section 4.

Theorem 1.3 Let $\mathcal{F}:=\left\{f_{1}, \ldots, f_{n}\right\} \subseteq \mathbb{C}\left[\mathbf{z}^{ \pm 1}\right]$ be a generic family of Laurent polynomials. Let $K$ be a connected component of $\mathcal{I}(\mathcal{F})$, let $P_{K}:=\operatorname{conv}(K)$ be the corresponding intersection polytope and let $P:=\operatorname{conv}(\mathcal{I}(\mathcal{F}))$. Then the following hold:

(a) There exists a generalized order map from the vertices of $P_{K}$ and $P$, respectively, to $\left(\operatorname{New}\left(f_{1}\right) \times\right.$ $\left.\cdots \times \operatorname{New}\left(f_{n}\right)\right) \cap \mathbb{Z}^{n \times n}$, that is injective on $P_{K}$ and on $P$, respectively.

(b) The vertices of $P$ are in 1-to-1-correspondence with those vertices of the Minkowski sum $\operatorname{New}\left(f_{1}\right)+$ $\cdots+\operatorname{New}\left(f_{n}\right)$, which have a mixed normal cone.

We omit the proofs of our results and refer to the preprint Juhnke-Kubitzke and de Wolff (2015) for this.

\section{Preliminaries}

\subsection{Polytopes and their Normal Fans}

Throughout this article, we assume that the reader is familiar with basic objects from discrete geometry. For background information we recommend Ziegler (2007) as a reference. We remind the reader that 
for a given a Laurent polynomial $f:=\sum_{a \in \mathbb{Z}^{n}} \lambda_{a} \mathbf{z}^{a} \in \mathbb{C}\left[\mathbf{z}^{ \pm 1}\right]$, where $\mathbf{z}^{a}:=z_{1}^{a_{1}} \cdots z_{n}^{a_{n}}$ for $a:=$ $\left(a_{1}, \ldots, a_{n}\right) \in \mathbb{Z}^{n}$, its Newton polytope $\operatorname{New}(f)$ is the lattice polytope defined by

$$
\operatorname{New}(f):=\operatorname{conv}\left\{a \in \mathbb{Z}^{n}: \lambda_{a} \neq 0\right\} \subseteq \mathbb{R}^{n} .
$$

In the following, the set $\left\{a: \lambda_{a} \neq 0\right\}$ will also be referred to as the support of $f$.

Given a polytope $P \subseteq \mathbb{R}^{n}$, we denote by $\mathrm{NF}_{G}(P)$ the normal cone corresponding to a non-empty face $G \in P$ The collection of the normal cones is called the normal fan of $P$, denoted by $\operatorname{NF}(P)$ : If $P:=\operatorname{New}(f)$ is the Newton polytope of a Laurent polynomial $f \in \mathbb{C}\left[\mathbf{z}^{ \pm 1}\right]$, then we write $\mathrm{NF}_{G}(f)$ for $\mathrm{NF}_{G}(\operatorname{New}(f))$, where $G$ is a face of $\operatorname{New}(f)$. Similarly, we write $\operatorname{NF}(f)$ for the normal fan of New $(f)$.

\subsection{The Order Map}

Given a Laurent polynomial $f \in \mathbb{C}\left[\mathbf{z}^{ \pm \mathbf{1}}\right]$, the complement of $\mathcal{A}(f)$ consists of several bounded or unbounded connected components. As shown in Forsberg et al. (2000), there exists a close connection between those components and lattice points in the corresponding Newton polytope $\operatorname{New}(f)$. The precise relation is given via the order map:

$$
\begin{aligned}
\text { ord }: \mathbb{R}^{n} \backslash \mathcal{A}(f) & \rightarrow \mathbb{R}^{n}, \quad \mathbf{w} \mapsto\left(u_{1}, \ldots, u_{n}\right) \text { with } \\
u_{j} & :=\frac{1}{(2 \pi i)^{n}} \int_{\log |\mathbf{z}|=\mathbf{w}} \frac{z_{j} \partial_{j} f(\mathbf{z})}{f(\mathbf{z})} \frac{d z_{1} \cdots d z_{n}}{z_{1} \cdots z_{n}} \text { for all } 1 \leq j \leq n .
\end{aligned}
$$

For simplicity, we write $\operatorname{im}(\operatorname{ord}(f))$ for the image of $\mathbb{R}^{n} \backslash \mathcal{A}(f)$ under the order map.

The following theorem from Forsberg et al. (2000) provides the indicated connection between components of the complement of $\mathcal{A}(f)$ and lattice points in $\operatorname{New}(f)$.

Theorem 2.1 Given a Laurent polynomial $f \in \mathbb{C}\left[\mathbf{z}^{ \pm 1}\right]$ the image of the order map $\operatorname{im}(\operatorname{ord}(f))$ is contained in $\operatorname{New}(f) \cap \mathbb{Z}^{n}$. If $\mathbf{w}, \mathbf{w}^{\prime} \in \mathbb{R}^{n} \backslash \mathcal{A}(f)$, then $\mathbf{w}$ and $\mathbf{w}^{\prime}$ belong to the same component of the complement of $\mathcal{A}(f)$ if and only if $\operatorname{ord}(\mathbf{w})=\operatorname{ord}\left(\mathbf{w}^{\prime}\right)$.

As a consequence of the previous theorem, every component of the complement of a given amoeba $\mathcal{A}(f)$ corresponds to a unique lattice point in the Newton polytope New $(f)$ of $f$. In the following, we denote for each $\alpha \in \operatorname{New}(f) \cap \mathbb{Z}^{n}$ its corresponding (possibly empty) component of the complement of $\mathcal{A}(f)$ by $E_{\alpha}(f)$, i.e., $E_{\alpha}(f):=\left\{\mathbf{w} \in \mathbb{R}^{n} \backslash \mathcal{A}(f): \operatorname{ord}(\mathbf{w})=\alpha\right\}$.

Points in $E_{\alpha}(f)$ are of order $\alpha$ and $E_{\alpha}(f)$ is called the component of order $\alpha$ of the complement.

Besides the connection between components of the complement of $\mathcal{A}(f)$ and lattice points in New $(f)$, it was shown in Gelfand et al. (1994) that the former ones are strongly linked to the normal fan of New $(f)$ :

Theorem 2.2 Let $f \in \mathbb{C}\left[\mathbf{z}^{ \pm 1}\right]$ with support set $A \subseteq \mathbb{Z}^{n}$. The set of vertices of $\operatorname{New}(f)$ is in bijective correspondence with a subset of the components of the complement of $\mathcal{A}(f)$. Namely, let $\alpha \in A$ be a vertex in $\mathrm{New}(f)$ and $\mathrm{NF}_{\alpha}(f)$ be the corresponding cone in $\mathrm{NF}(f)$. Then there exists a unique nonempty, unbounded component $E_{\alpha}(f)$ in the complement of $\mathcal{A}(f)$ that contains an affine translation of $\mathrm{NF}_{\alpha}(f)$. 


\subsection{Tropical Geometry and the Spine}

Looking at the image of an amoeba, one immediately observes that it has finitely many "tentacles", which point in different directions. These tentacles direct to a set of points at infinity, which is called the logarithmic limit set, see Bergman (1971). For a Laurent polynomial $f$ with amoeba $\mathcal{A}(f)$ and any positive real number $r \in \mathbb{R}$, one defines

$$
\mathcal{A}_{r}(f):=(1 / r \cdot \mathcal{A}(f)) \cap \mathbb{S}^{n} .
$$

Here, $1 / r \cdot \mathcal{A}(f):=\{1 / r \cdot \mathbf{w}: \mathbf{w} \in \mathcal{A}(f)\}$ and $\mathbb{S}^{n}:=\left\{\mathbf{w} \in \mathbb{R}^{n}:\|\mathbf{w}\|_{2}=1\right\}$. The logarithmic limit set $\mathcal{A}_{\infty}(f)$ is defined as $\mathcal{A}_{\infty}(f):=\lim _{r \rightarrow \infty} \mathcal{A}_{r}(f)$. It was shown in Bieri and Groves (1984) that $\mathcal{A}_{\infty}(f)$ is a rational, polyhedral fan on the unit sphere; see also Maclagan and Sturmfels (2015). Note also that amoebas $\mathcal{A}(f)$ are unbounded, and their complements are open. This fact will be used in Section 2.3

In the following, we introduce the spine of an amoeba $\mathcal{A}(f)$, which is a more sophisticated way to describe the latter one.

We need to recall some notion from tropical geometry. For background on tropical geometry we refer to Maclagan and Sturmfels (2015). The tropical semiring $(\mathbb{R} \cup\{-\infty\}, \oplus, \odot)$ is defined by the operations

$$
a \oplus b:=\max \{a, b\}, \quad \text { and } \quad a \odot b:=a+b .
$$

A tropical polynomial with support set $A \subseteq \mathbb{N}^{n}$ is a function

$$
(\mathbb{R} \cup\{-\infty\})^{n} \rightarrow \mathbb{R} \cup\{-\infty\}, \quad\left(z_{1}, \ldots, z_{n}\right) \mapsto \bigoplus_{a \in A} b_{a} \odot \mathbf{z}^{a}:=\max _{a \in A}\left\{b_{a}+\langle\mathbf{z}, a\rangle\right\},
$$

where $b_{a} \in \mathbb{R} \backslash\{0\}$. For a tropical polynomial $h$, its tropical hypersurface $\mathcal{T}(h)$ is defined as the set of points $\mathbf{x}$ in $(\mathbb{R} \cup\{-\infty\})^{n}$ such that the maximum of $\left\{b_{a}+\langle\mathbf{x}, a\rangle: a \in A\right\}$ is attained at least twice. The tropical hypersurface $\mathcal{T}(h)$ is a polyhedral complex, which is dual to a subdivision of the Newton polytope of $h$.

The definition of the spine of an amoeba requires the definition of the Ronkin function Ronkin (2001). Let $\Omega$ be a convex open set in $\mathbb{R}^{n}$ and let $f \in \mathbb{C}\left[\mathbf{z}^{ \pm 1}\right]$, which is defined on $\log ^{-1}|\Omega|$. The Ronkin function $R_{f}: \Omega \rightarrow \mathbb{R}$ satisfies the following properties.

Theorem 2.3 (Ronkin (2001) / Passare and Rullgård (2004)) Let $f \in \mathbb{C}\left[\mathbf{z}^{ \pm 1}\right]$ be a holomorphic function. Then $R_{f}$ is a convex function. If $U \subseteq \Omega$ is a connected open set, then the restriction of $R_{f}$ to $U$ is affine linear if and only if $U \cap \mathcal{A}(f)=\emptyset$. If $\mathbf{x}$ is in the complement of $\mathcal{A}(f)$, then the gradient of $R_{f}(\mathbf{x})$ equals the order of $\mathbf{x}$.

Given a Laurent polynomial $f$ and a point $\alpha$ in its support set, we have seen in Section 2.2 that the corresponding component $E_{\alpha}(f)$ of the complement of $\mathcal{A}(f)$ is non-empty if and only if $\alpha \in \operatorname{im}(\operatorname{ord}(f))$. For every $\alpha \in \operatorname{im}(\operatorname{ord}(f))$ one defines the Ronkin coefficient of $\alpha$ by

$$
r_{\alpha}:=R_{f}(\mathbf{x})-\langle\alpha, \mathbf{x}\rangle \text { for every } \mathbf{x} \in E_{\alpha}(f) .
$$

Note that, due to Theorem 2.3, $r_{\alpha}$ is well-defined. The Ronkin coefficients give rise to the following tropical polynomial

$$
\operatorname{SpineT}(f):=\bigoplus_{\alpha \in \operatorname{im}(\operatorname{ord}(f))} r_{\alpha} \oplus \mathbf{x}^{\alpha}
$$


The tropical hypersurface given by $\operatorname{SpineT}(f)$ is called the spine of $f$, which is denoted by $\mathcal{S}(f)$. I.e., $\mathcal{S}(f):=\mathcal{T}(\operatorname{SpineT}(f))$. It was shown in Passare and Rullgård (2004) that the spine of a Laurent polynomial $f \in \mathbb{C}\left[\mathbf{z}^{ \pm 1}\right]$ is a deformation retract of $\mathcal{A}(f)$.

\subsection{Mixed Volumes and the Bernstein Theorem}

Following (Gelfand et al., 1994, p. 205) we provide the following definition of mixed volumes. Let $P_{1}, \ldots, P_{n}$ be (convex) polytopes in $\mathbb{R}^{n}$ and for $\lambda \in \mathbb{R}$ let $\lambda P_{i}:=\{\lambda \mathbf{p}: \mathbf{p} \in P\}$. Given a translationinvariant volume form $\operatorname{Vol}$ on $\mathbb{R}^{n}$, the expression $\operatorname{Vol}\left(\lambda_{1} P_{1}+\cdots+\lambda_{n} P_{n}\right)$ is a homogeneous polynomial in $\lambda_{1}, \ldots, \lambda_{n}$ of degree $n$.

Definition 2.4 The mixed volume $\operatorname{Vol}\left(P_{1}, \ldots, P_{n}\right)$ is the coefficient of the monomial $\lambda_{1} \cdots \lambda_{n}$ in the polynomial $\operatorname{Vol}\left(\lambda_{1} P_{1}+\cdots+\lambda_{n} P_{n}\right)$, i.e.,

$$
\operatorname{Vol}\left(P_{1}, \ldots, P_{n}\right):=\frac{1}{n !} \sum_{j=1}^{n}(-1)^{n-j} \sum_{1 \leq i_{1}<\cdots<i_{j} \leq n} \operatorname{Vol}\left(P_{i_{1}}+\cdots+P_{i_{j}}\right) .
$$

Since in the sequel, we will always consider lattice polytopes, we choose MV as the volume form that is induced by the lattice $\mathbb{Z}^{n}$ and that satisfies that every standard simplex has volume 1; see (Gelfand et al. 1994. Chapter 5, Section 3D). The classical Bernstein Theorem states the following; see Bernstein (1975) and (Gelfand et al., 1994, Theorem 2.8., p. 206):

Theorem 2.5 (Bernstein Theorem) Let $A_{1}, \ldots, A_{n} \subset \mathbb{Z}^{n}$ be finite sets such that their union generates $\mathbb{Z}^{n}$ as an affine lattice. Let $P_{i} \subseteq \mathbb{R}^{n}$ be the convex hull of $A_{i}$, and let $\mathbb{C}^{A_{i}}$ be the space of Laurent polynomials with support set $A_{i}$. Then there exists a dense Zariski open subset $U \subseteq \mathbb{C}^{A_{1}} \times \cdots \times \mathbb{C}^{A_{n}}$ satisfying: for any $\left(f_{1}, \ldots, f_{n}\right) \in U$, the number of solutions of the system of equations $f_{1}(\mathbf{z})=\cdots=$ $f_{n}(\mathbf{z})=0$ in $\left(\mathbb{C}^{*}\right)^{n}$ equals the mixed volume $\operatorname{MV}\left(P_{1}, \ldots, P_{n}\right)$.

Let $P_{1}, \ldots, P_{n}$ be lattice $n$-polytopes and let $P:=P_{1}+\cdots+P_{n}$. A sum $C:=F_{1}+\cdots+F_{n}$, where $F_{i} \subseteq P_{i}$ is a face $(1 \leq i \leq n)$, is called a cell of $P$. A subdivision of $P$ is a collection $\Gamma:=\left\{C_{1}, \ldots, C_{m}\right\}$ of cells such that each cell is of full dimension, the intersection of two cells is a face of both and the union of all cells covers $P$. A subdivision $\Gamma$ is called mixed if for each cell $C=F_{1}+\cdots+F_{n} \in \Gamma$ one has $n=\operatorname{dim} F_{1}+\cdots+\operatorname{dim} F_{n}$. A cell $C$ is called mixed if every $P_{i}$ contributes with a face of dimension at least 1 to $C$. For further information and the following statement see Steffens and Theobald (2010).

Lemma 2.6 Let $P_{1}, \ldots, P_{n}$ be lattice n-polytopes and let $\Gamma$ be an arbitrary mixed subdivision of $P:=$ $P_{1}+\cdots+P_{n}$. Then we have $\operatorname{MV}\left(P_{1}, \ldots, P_{n}\right)=\sum_{C \text { mixed cell in } \Gamma} \operatorname{MV}(C)$.

An intersection of $k$ tropical hypersurfaces in $\mathbb{R}^{n}$ is called proper if it has codimension $n-k$. Any $\varepsilon$ perturbation of an arbitrary intersection of $k$ tropical hypersurfaces becomes transversal. Given $k$ tropical hypersurfaces their stable intersection is defined as the limit, for $\varepsilon \rightarrow 0$, of an $\varepsilon$-perturbation of the original (possibly non-transversal) intersection. Every stable intersection is proper; see e.g., (Maclagan and Sturmfels, 2015, Section 3.6.) for further details.

The next theorem is atropical analog of the Bernstein Theorem (see (Maclagan and Sturmfels, 2015. Theorem 4.6.9., p. 196)).

Theorem 2.7 (Tropical Bernstein Theorem) Let $\mathcal{T}\left(h_{1}\right), \ldots, \mathcal{T}\left(h_{n}\right) \in \mathbb{R}^{n}$ be generic tropical hypersurfaces which are dual to regular subdivisions of $\operatorname{New}\left(h_{1}\right), \ldots, \operatorname{New}\left(h_{n}\right) \subseteq \mathbb{R}^{n}$. The cardinality of their stable intersection $\mathcal{T}\left(h_{1}\right) \cap \cdots \cap \mathcal{T}\left(h_{n}\right)$ equals the mixed volume $\operatorname{MV}\left(\operatorname{New}\left(h_{1}\right), \ldots, \operatorname{New}\left(h_{n}\right)\right)$. 


\section{Combinatorics of Intersections of Amoebas}

In this section we study basic combinatorial properties of intersections of hypersurface amoebas.

We start by fixing some notation. In the following, we will always assume that $n \geq 2$. Throughout this article, we will call a family $\mathcal{F}:=\left\{f_{1}, \ldots, f_{n}\right\} \subseteq \mathbb{C}\left[\mathbf{z}^{ \pm 1}\right]$ of Laurent polynomials generic if the following conditions hold:

(1) every $f_{j} \in \mathcal{F}$ is irreducible,

(2) for every $1 \leq k \leq n$ and every $k$-element subset $\left\{f_{i_{1}}, \ldots, f_{i_{k}}\right\} \subseteq \mathcal{F}$ the intersection $\bigcap_{\ell=1}^{k} \partial\left(\mathcal{A}\left(f_{i_{\ell}}\right)\right)$ has codimension $k$,

(3) for every $1 \leq k \leq n$ and every $k$-element subset $\left\{f_{i_{1}}, \ldots, f_{i_{k}}\right\} \subseteq \mathcal{F}$ the intersection of the corresponding spines $\mathcal{S}\left(f_{i_{1}}\right), \ldots, \mathcal{S}\left(f_{i_{k}}\right)$ is stable and hence of codimension $k$.

(4) for every $1 \leq j \leq n$ and for every $p \in \partial\left(\mathcal{A}\left(f_{j}\right)\right)$, there exists a unique component of the complement of $\mathcal{A}\left(f_{j}\right)$ containing $p$ in its closure.

Moreover, a single Laurent polynomial $f$ is generic, if it satisfies condition (1) and (4).

Note that this kind of genericity implies that the boundaries of all amoebas in $\mathcal{F}$ have a non-trivial intersection. Hence, it cannot happen that $\mathcal{A}\left(f_{i}\right) \subseteq \mathcal{A}\left(f_{j}\right)$ for some $1 \leq i, j \leq n$, where $i \neq j$.

In the following, we consider a generic family $\mathcal{F}:=\left\{f_{1}, \ldots, f_{n}\right\} \subseteq \mathbb{C}\left[\mathbf{z}^{ \pm 1}\right]$ of Laurent polynomials.We are interested in the intersection $\mathcal{I}(\mathcal{F}):=\bigcap_{j=1}^{n} \mathcal{A}\left(f_{j}\right)$. An initial example shows that, in general, $\mathcal{I}(\mathcal{F})$ is disconnected.

Example 3.1 Let $\mathcal{F}:=\left\{f_{1}, f_{2}\right\} \subseteq \mathbb{C}\left[z_{1}^{ \pm 1}, z_{2}^{ \pm 1}\right]$, where $f_{1}\left(z_{1}, z_{2}\right):=2 z_{1}+z_{2}+1$ and $f_{2}\left(z_{1}, z_{2}\right):=$ $z_{1}^{2} z_{2}+z_{1} z_{2}^{2}+5 z_{1} z_{2}+1$. One can see from Figure 1 that $\mathcal{A}\left(f_{1}\right) \cap \mathcal{A}\left(f_{2}\right)$ consists of two disjoint connected components.

The next theorem states some basic properties of the connected components of $\mathcal{I}(\mathcal{F})$.

Theorem 3.2 Let $n \geq 2$ and let $\mathcal{F}:=\left\{f_{1}, \ldots, f_{n}\right\} \subseteq \mathbb{C}\left[\mathbf{z}^{ \pm 1}\right]$ be generic. Then every connected component of $\mathcal{I}(\mathcal{F})$ is a compact set of dimension $n$.

In order to obtain information about the connected components of the intersection $\mathcal{I}(\mathcal{F})$ of $\mathcal{A}\left(f_{1}\right), \ldots, \mathcal{A}\left(f_{n}\right)$, it is important to understand the boundaries of those components. The following simple lemma is a direct consequence of our notion of genericity, defined at the beginning of this section.

Lemma 3.3 Let $\mathcal{F}:=\left\{f_{1}, \ldots, f_{k}\right\} \subset \mathbb{C}\left[\mathbf{z}^{ \pm 1}\right]$ be a generic collection of Laurent polynomials. Then $\bigcap_{j=1}^{k} \partial\left(\mathcal{A}\left(f_{j}\right) \backslash \mathcal{A}_{\infty}\left(f_{j}\right)\right)$ is of dimension $n-k$. Particularly, if $k=n$, then the intersection is zero dimensional and of finite cardinality.

Note that it is immediate from Lemma 3.3, that in our situation the intersection of the boundaries of $\mathcal{A}\left(f_{1}\right), \ldots, \mathcal{A}\left(f_{n}\right)$ is always a finite point set. This motivates the following definition.

Definition 3.4 Let $\mathcal{F}:=\left\{f_{1}, \ldots f_{n}\right\} \subseteq \mathbb{C}\left[\mathbf{z}^{ \pm 1}\right]$ be a generic collection of Laurent polynomials.

(a) $\bigcap_{j=1}^{n} \partial\left(\mathcal{A}\left(f_{j}\right)\right)$ is called the set of vertices of $\mathcal{I}(\mathcal{F})$, denoted by $V(\mathcal{F})$.

(b) For a connected component $K$ of $\mathcal{I}(\mathcal{F})$ we call $V(K):=K \cap V(\mathcal{F})$ the set of vertices of $K$. 


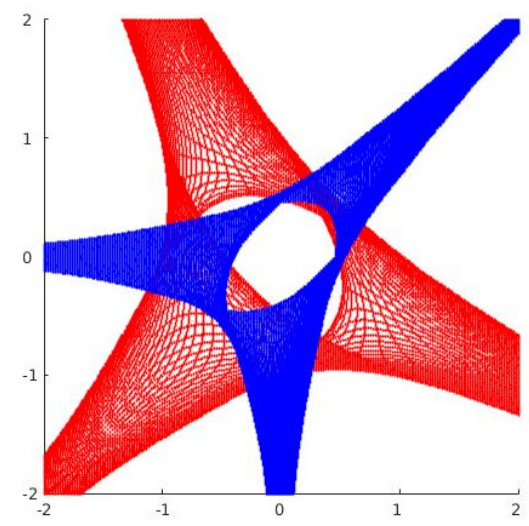

Fig. 2: The intersection of $f_{1}\left(z_{1}, z_{2}\right):=z_{1}^{2} z_{2}^{2}+z_{1} z_{2}+z_{1}+z_{2}$ and $f_{2}\left(z_{1}, z_{2}\right):=z_{1}^{3}+z_{2}^{3}+2 z_{1} z_{2}+1$. None of the vertices of $\mathcal{I}\left(\left\{f_{1}, f_{2}\right\}\right)$ given by the intersection of the boundaries of the two bounded components of the complement of $\mathcal{A}\left(f_{1}\right)$ and $\mathcal{A}\left(f_{2}\right)$ are vertices of the corresponding intersection polytopes.

By Definition 3.4 vertices of a connected component $K$ of $\mathcal{I}(\mathcal{F})$ lie on the boundary of $K$. More generally, we can decompose the boundary of $K$ as the union of disjoint open pieces, each of which is contained in the intersection of finitely many $\partial \overline{E_{\alpha_{j}}\left(f_{j}\right)}$. This motivates the following definition of $k$-faces of an $K$.

Definition 3.5 Let $\mathcal{F}:=\left\{f_{1}, \ldots f_{n}\right\} \subseteq \mathbb{C}\left[\mathbf{z}^{ \pm 1}\right]$ be a generic collection of Laurent polynomials. Let $K$ be a connected component of $\mathcal{I}(\mathcal{F})$. Let $0 \leq k \leq n-1$.

(a) A non-empty and connected subset $F \subsetneq K$ is called a $k$-face of $K$, if there exist unique $E_{\alpha_{1}}\left(f_{j_{1}}\right), \ldots, E_{\alpha_{n-k}}\left(f_{j_{n-k}}\right)$ such that $\mathbf{x} \in \bigcap_{s=1}^{n-k} \partial \overline{E_{\alpha_{s}}\left(f_{j_{s}}\right)}$ for all $\mathbf{x} \in F$.

(b) An $(n-1)$-dimensional face of $K$ is called a facet of $K$.

Note that a priori the definition of a face does not exclude that a single amoeba contributes with multiple components of its complement to an intersection that describes a specific face. The following lemma, however, shows that this case can never occur.

Lemma 3.6 Let $\mathcal{F}:=\left\{f_{1}, \ldots f_{n}\right\} \subseteq \mathbb{C}\left[\mathbf{z}^{ \pm 1}\right]$ be generic, $K$ be a connected component of $\mathcal{I}(\mathcal{F})$, and $F$ be a $k$-face of $K$ given by $E_{\alpha\left(l_{1}\right)}\left(f_{j_{1}}\right), \ldots, E_{\alpha\left(l_{n-k}\right)}\left(f_{j_{n-k}}\right)$. Then all the $f_{j_{s}}$ are distinct.

Given a connected component $K$ of $\mathcal{I}(\mathcal{F})$ with set of vertices $V(K)$, we define the polytope $P_{K}$ as the convex hull of $V(K)$. All polytopes arising in this way will be called intersection polytopes of $\mathcal{F}$. We remark that though the vertex set of an intersection polytope $P_{K}$ is contained in $V(K)$, it does not have to coincide with $V(K)$; see Figure 2 The following theorem shows that the vertex set $V\left(P_{K}\right)$ of $P_{K}$ coincides with the set of extreme points of $\operatorname{conv}(K)$.

Theorem 3.7 Let $\mathcal{F}:=\left\{f_{1}, \ldots, f_{n}\right\} \subseteq \mathbb{C}\left[\mathbf{z}^{ \pm 1}\right]$ be generic and let $K$ be a connected component of $\mathcal{I}(\mathcal{F})$. Then the vertex set $V\left(P_{K}\right)$ of $P_{K}$ is given by the set of extreme points of $\operatorname{conv}(K)$. Particularly, we have $\operatorname{conv}(K)=P_{K}$. 
For intersection polytopes the following statement holds.

Proposition 3.8 Let $\mathcal{F}:=\left\{f_{1}, \ldots, f_{n}\right\} \subseteq \mathbb{C}\left[\mathbf{z}^{ \pm 1}\right]$ be a generic collection of Laurent polynomials and let $K$ be a connected component of $\mathcal{I}(\mathcal{F})$. Then, the intersection polytope $P_{K}$ is simple.

Let $\mathcal{F}:=\left\{f_{1}, \ldots, f_{n}\right\} \subseteq \mathbb{C}\left[\mathbf{z}^{ \pm 1}\right]$. Since a spine is a deformation retract of its corresponding amoeba, it is natural to ask if the different connected components of an intersection $\mathcal{I}(\mathcal{F})$ can already be detected from the intersection of the spines of $\mathcal{A}\left(f_{1}\right), \ldots, \mathcal{A}\left(f_{n}\right)$. On the one hand, if the spines have a common intersection, then this gives rise to a connected component of $\mathcal{I}(\mathcal{F})$. On the other hand, it is not clear a priori whether every connected component of $\mathcal{I}(\mathcal{F})$ contains a common point of intersection of the spines of $\mathcal{A}\left(f_{1}\right), \ldots, \mathcal{A}\left(f_{n}\right)$.

The next theorem answers this question in the affirmative.

Theorem 3.9 Let $\mathcal{F}:=\left\{f_{1}, \ldots, f_{n}\right\} \subseteq \mathbb{C}\left[\mathbf{z}^{ \pm 1}\right]$ be a generic collection of Laurent polynomials and let $K$ be a connected component of $\mathcal{I}(\mathcal{F})$. Then $K \cap \bigcap_{j=1}^{n} \mathcal{S}\left(f_{j}\right) \neq \emptyset$. Particularly, the number of connected components of $\mathcal{I}(\mathcal{F})$ is at most $\# \bigcap_{j=1}^{n} \mathcal{S}\left(f_{j}\right)$.

As consequence of the previous theorem we obtain the Amoeba Bernstein Theorem.

Amoeba Bernstein Theorem 3.10 Let $\mathcal{F}:=\left\{f_{1}, \ldots, f_{n}\right\} \subseteq \mathbb{C}\left[\mathbf{z}^{ \pm 1}\right]$ be a generic collection of Laurent polynomials. Let $c$ be the number of connected components of $\mathcal{I}(\mathcal{F})$. Then $c$ is bounded from above by the number of mixed cells in the subdivision of $\mathrm{New}\left(f_{1}\right)+\cdots+\operatorname{New}\left(f_{n}\right)$, which is induced by the subdivisions of $\operatorname{New}\left(f_{1}\right), \ldots, \operatorname{New}\left(f_{n}\right)$ that are dual to the spines $\mathcal{S}\left(f_{i}\right), \ldots, \mathcal{S}\left(f_{n}\right)$. Particularly, c is bounded from above by the mixed volume $\operatorname{MV}\left(\operatorname{New}\left(f_{1}\right), \ldots, \operatorname{New}\left(f_{n}\right)\right)$.

As a corollary of Theorem 3.9 we get the following Bézout type statement for the intersection of amoebas.

Amoeba Bézout Theorem 3.11 Let $\mathcal{F}:=\left\{f_{1}, \ldots, f_{n}\right\} \subseteq \mathbb{C}\left[\mathbf{z}^{ \pm 1}\right]$ be a generic collection of Laurent polynomials. For $1 \leq j \leq n$, let $d_{j}:=\operatorname{deg}\left(\operatorname{trop}_{S}\left(f_{j}\right)\right)$. Let $c$ be the number of connected components of $\mathcal{I}(\mathcal{F})$. Then, $c \leq \prod_{j=1}^{n} d_{j}$. Particularly, $c \leq \prod_{j=1}^{n} \operatorname{deg}\left(f_{j}\right)$.

\section{A Generalized Order Map}

In this section, we generalize the order map of an amoeba to intersections of generic collections of amoebas. So as to obtain a well-defined map it is necessary to assume that if a polynomial $f$ belongs to a generic collection $\mathcal{F} \subseteq \mathbb{C}\left[\mathbf{z}^{ \pm \mathbf{1}}\right]$, then for any point $p$ on the boundary of $\mathcal{A}(f)$ there exists a unique component of the complement of $\mathcal{A}(f)$ containing $p$ in its closure; see condition (4) in our definition of "generic". We remark that this is not for all boundary points of all amoebas the case; see (Rullgård 2003, Figure 2, p. 58) for an example. However, if $f$ is generic, then the order map can be extended to the boundary of an amoeba. Namely, if $p \in \overline{E_{\alpha}(f)} \cap \partial \mathcal{A}(f)$, then we define $\operatorname{ord}(p):=\alpha$, i.e., the order of a point $p$ on the boundary of an amoeba $\mathcal{A}(f)$ is the order of the unique component of the complement, which contains $p$ in its closure. From now on assume that $\mathcal{F}:=\left\{f_{1}, \ldots, f_{n}\right\} \subseteq \mathbb{C}\left[\mathbf{z}^{ \pm 1}\right]$ is a generic collection of Laurent polynomials. For $1 \leq i \leq n$, we use ord $i$ to denote the just described extension of the order map of $f_{i}$. In order to simplify the notation further, we write $\operatorname{New}(\mathcal{F})$ for the Cartesian product of the Newton polytopes of $f_{1}, \ldots, f_{n}$, i.e., $\operatorname{New}(\mathcal{F}):=\operatorname{New}\left(f_{1}\right) \times \cdots \times \operatorname{New}\left(f_{n}\right)$. 
Given these prerequisites, we generalize the order map of amoebas to intersections of amoebas in the following way: The generalized order map of $\mathcal{F}$ is defined by

$$
\operatorname{ord}_{\mathcal{F}}: V(\mathcal{F}) \rightarrow \mathbb{Z}^{n \times n} \quad p \mapsto\left(\operatorname{ord}_{1}(p), \ldots, \operatorname{ord}_{n}(p)\right)^{T} .
$$

We refer to $\operatorname{ord}_{\mathcal{F}}(p)$ as the order matrix of $p \in V(\mathcal{F})$. Though the generalized order map ord $\operatorname{Fi}_{\mathcal{F}}$ is not injective in general, it is injective if one restricts oneselve to a single intersection polytope.

Theorem 4.1 Let $\mathcal{F}:=\left\{f_{1}, \ldots, f_{n}\right\} \subseteq \mathbb{C}\left[\mathbf{z}^{ \pm 1}\right]$ be a generic collection of Laurent polynomials. Let $K$ be a connected component of $\mathcal{I}(\mathcal{F})$, and let $P_{K}$ be the corresponding intersection polytope. Let $p$ and $q$ be vertices of $P_{K}$. Then $\operatorname{ord}_{\mathcal{F}}(p) \neq \operatorname{ord}_{\mathcal{F}}(q)$. Particularly, $\operatorname{ord}_{\mathcal{F}}$ is injective on $V\left(P_{K}\right)$.

Though, the generalized order map is injective on each intersection polytope, this does not have to be true if one considers restrictions of the generalized order map to the vertex set of a connected component of $\mathcal{I}(\mathcal{F})$. For instance, in 2 , both connected components have two vertices, that are not vertices of the corresponding intersection polytopes, whose orders are equal. It follows from the proof of Theorem 4.1 that if $K$ is a connected component of $\mathcal{I}(\mathcal{F})$ and if $\operatorname{ord}(p)=\operatorname{ord}(q)$ for two vertices $p, q \in V(K)$, then neither $p$ nor $q$ can be vertices of the corresponding intersection polytope $P_{K}$.

The next proposition describes the normal cones of specific vertices of an intersection polytope.

Proposition 4.2 Let $\mathcal{F}:=\left\{f_{1}, \ldots, f_{n}\right\} \subseteq \mathbb{C}\left[\mathbf{z}^{ \pm 1}\right]$ be a generic collection of Laurent polynomials. Let $K$ be a connected component of $\mathcal{I}(\mathcal{F})$ and $P_{K}$ be the corresponding intersection polytope. Let $p \in V\left(P_{K}\right)$ be a vertex of $P_{K}$ such that $\operatorname{ord}_{i}(p)$ corresponds to a vertex $v_{i}$ of $\operatorname{New}\left(f_{i}\right)$ for $1 \leq i \leq n$. Then the normal cone of $p$ in $P_{K}$ contains an affine translation of the intersection of all normal cones of the $v_{i}$ in $\operatorname{New}\left(f_{i}\right)$ for $1 \leq i \leq n$, i.e., there exists a $v \in \mathbb{R}^{n}$ such that

$$
v+\bigcap_{i=1}^{n} \mathrm{NF}_{v_{i}}\left(f_{i}\right) \subseteq \mathrm{NF}_{p}\left(P_{K}\right) .
$$

Since there is a relation between components of the complement of a single amoeba and the vertices of the corresponding Newton polytope, it is reasonable to ask if such a relation also exists if one considers intersections of amoebas and the Minkowski sum of their Newton polytopes. For given Newton polytopes $\operatorname{New}\left(f_{1}\right), \ldots, \operatorname{New}\left(f_{n}\right)$ one defines the common refinement $\operatorname{NF}\left(f_{1}, \ldots, f_{n}\right)$ of the normal fans $\mathrm{NF}\left(f_{1}\right), \ldots, \mathrm{NF}\left(f_{n}\right)$ as the fan given by all cones of the form $\bigcap_{j=1}^{n} \mathrm{NF}_{G_{j}}\left(f_{j}\right)$, where $\mathrm{NF}_{G_{j}}\left(f_{j}\right)$ is an arbitrary cone of $\mathrm{NF}\left(f_{j}\right)$.

Definition 4.3 We call a non-empty, full dimensional cone $\bigcap_{j=1}^{n} \mathrm{NF}_{G_{j}}\left(f_{j}\right)$ in the common refinement $\mathrm{NF}\left(f_{1}, \ldots, f_{n}\right)$ mixed if it satisfies for all $1 \leq i<j \leq n$ :

$$
\mathrm{NF}_{G_{i}}\left(f_{i}\right) \cap \bigcap_{j \in[n] \backslash\{i\}} \mathrm{NF}_{G_{j}}\left(f_{j}\right) \neq \mathrm{NF}_{G_{i}}\left(f_{i}\right), \bigcap_{j \in[n] \backslash\{i\}} \mathrm{NF}_{G_{j}}\left(f_{j}\right) .
$$

Since we are aiming at a possible connection between $\mathcal{I}(\mathcal{F})$ and the Minkowski sum of $\operatorname{New}\left(f_{1}\right)$, $\ldots, \operatorname{New}\left(f_{n}\right)$, we consider not only a single intersection polytope but the convex hull $\operatorname{conv}(V(\mathcal{F}))$ of all intersection polytopes. It is an obvious question, which points in $V(\mathcal{F})$ are vertices of $\operatorname{conv}(V(\mathcal{F}))$. We are able to provide a characterization of the vertex set of $\operatorname{conv}(V(\mathcal{F}))$ in terms of vertices of the Minkowski sum $\operatorname{New}\left(f_{1}\right)+\cdots+\operatorname{New}\left(f_{n}\right)$ alone. 
Theorem 4.4 Let $\mathcal{F}:=\left\{f_{1}, \ldots, f_{n}\right\} \subseteq \mathbb{C}\left[\mathbf{z}^{ \pm 1}\right]$ be a generic collection of Laurent polynomials. Let $p \in V(\mathcal{F})$. Then $p$ is a vertex of $\operatorname{conv}(V(\mathcal{F}))$ if and only if the following holds:

For $1 \leq j \leq n$ there exists a vertex $v:=v_{1}+\cdots+v_{n} \in \operatorname{New}\left(f_{1}\right)+\cdots+\operatorname{New}\left(f_{n}\right)$, where $v_{j} \in \operatorname{New}\left(f_{j}\right)$ is a vertex, such that $p \in \bigcap_{j=1}^{n} \partial\left(\overline{E_{v_{j}}\left(f_{j}\right)}\right)$ and $\mathrm{NF}_{v}\left(\operatorname{New}\left(f_{1}\right)+\cdots+\operatorname{New}\left(f_{n}\right)\right)$ is a mixed cone.

One can see from the proof of this theorem that the intersection $\bigcap_{j=1}^{n} \partial\left(\overline{E_{v_{j}}\left(f_{j}\right)}\right)$ equals a single point, which is a vertex of $\operatorname{conv}(V(\mathcal{F}))$. Now, the following is an immediate consequence of Theorem 4.4 .

Corollary 4.5 Let $\mathcal{F}:=\left\{f_{1}, \ldots, f_{n}\right\} \subseteq \mathbb{C}\left[\mathbf{z}^{ \pm 1}\right]$ be a generic collection of Laurent polynomials. Let $P$ be the convex hull of the vertices of $\mathcal{I}(\overline{\mathcal{F}})$, i.e., $P:=\operatorname{conv}(V(\mathcal{F}))$ and let $V(P)$ be the vertex set of $P$. Then, the restriction of the order map $\operatorname{ord}_{\mathcal{F}}$ to $V(P)$ is injective.

\section{References}

G. Bergman. The logarithmic limit-set of an algebraic variety. Trans. Amer. Math. Soc., 157:459-469, 1971.

D. N. Bernstein. The number of roots of a system of equations. Funkcional. Anal. i Priložen., 9(3):1-4, 1975.

R. Bieri and J. Groves. The geometry of the set of characters induced by valuations. J. Reine Angew. Math., 347:168-195, 1984.

T. de Wolff. Amoebas and their tropicalizations - a survey. Preprint, 2015.

M. Forsberg, M. Passare, and A. Tsikh. Laurent determinants and arrangements of hyperplane amoebas. Adv. Math., 151:45-70, 2000.

I. Gelfand, M. Kapranov, and A. Zelevinsky. Discriminants, Resultants and Multidimensional Determinants. Birkhäuser, Boston, 1994.

S. Iliman and T. de Wolff. Amoebas, nonnegative polynomials and sums of squares supported on circuits, 2014. To appear in Res. Math. Sci.; see also arXiv:1402.0462.

M. Juhnke-Kubitzke and T. de Wolff. Intersections of amoebas. http://arxiv.org/abs/1510.08416, 2015.

R. Kenyon, A. Okounkov, and S. Sheffield. Dimers and amoebae. Ann. of Math. (2), 163(3):1019-1056, 2006.

D. Maclagan and B. Sturmfels. Introduction to Tropical Geometry. Amer. Math. Soc., Providence, R.I., 2015.

G. Mikhalkin. Real algebraic curves, the moment map and amoebas. Ann. Math., 151:309-326, 2000.

G. Mikhalkin. Amoebas of algebraic varieties and tropical geometry. In S. K. Donaldson, Y. Eliashberg, and M. Gromov, editors, Different Faces of Geometry, pages 257-300. Kluwer, New York, 2004.

M. Nisse. Amoeba finite basis does not exist in general. Preprint, arXiv:1403.3912, 2014. 
M. Passare and H. Rullgård. Amoebas, Monge-Ampére measures and triangulations of the Newton polytope. Duke Math. J., 121(3):481-507, 2004.

M. Passare, D. Pochekutov, and A. Tskih. Amoebas of complex hypersurfaces in statistical thermodynamics. Math. Phys. Anal. Geom., 15, 2012.

K. Purbhoo. A Nullstellensatz for amoebas. Duke Math. J., 141(3):407-445, 2008.

L. Ronkin. On zeros of almost periodic functions generated by functions holomorphic in a multicircular domain. In Complex Analysis in Modern Mathematics (Russian), pages 239-251. FAZIS, Moscow, 2001.

H. Rullgård. Topics in Geometry, Analysis and Inverse Problems. PhD thesis, Stockholm University, 2003.

F. Schroeter and T. de Wolff. The boundary of amoebas. Preprint, arXiv:1310.7363, 2013.

R. Steffens and T. Theobald. Combinatorics and genus of tropical intersections and Ehrhart theory. SIAM J. Discrete Math., 24(1):17-32, 2010.

G. Ziegler. Lectures on Polytopes. Springer Verlag, 2007. 